University of Nebraska - Lincoln

DigitalCommons@University of Nebraska - Lincoln

\title{
When Quantity Trumps Number: Discrimination Experiments in Cotton-Top Tamarins (Saguinus oedipus) and Common Marmosets (Callithrix jacchus)
}

\author{
Jeffrey R. Stevens \\ University of Nebraska-Lincoln, jstevens5@unl.edu \\ Justin N. Wood \\ Harvard University, justin.wood@usc.edu \\ Marc D. Hauser \\ Harvard University
}

Follow this and additional works at: https://digitalcommons.unl.edu/psychfacpub

Part of the Psychiatry and Psychology Commons

Stevens, Jeffrey R.; Wood, Justin N.; and Hauser, Marc D., "When Quantity Trumps Number: Discrimination Experiments in Cotton-Top Tamarins (Saguinus oedipus) and Common Marmosets (Callithrix jacchus)" (2007). Faculty Publications, Department of Psychology. 539.

https://digitalcommons.unl.edu/psychfacpub/539

This Article is brought to you for free and open access by the Psychology, Department of at DigitalCommons@University of Nebraska - Lincoln. It has been accepted for inclusion in Faculty Publications, Department of Psychology by an authorized administrator of DigitalCommons@University of Nebraska - Lincoln. 


\title{
When Quantity Trumps Number: Discrimination Experiments in Cotton-Top Tamarins (Saguinus oedipus) and Common Marmosets (Callithrix jacchus)
}

\author{
Jeffrey R. Stevens, ${ }^{1,4}$ Justin N. Wood, $^{1}$ and Marc D. Hauser ${ }^{1,2,3}$ \\ 1. Cognitive Evolution Laboratory, Department of Psychology, Harvard University, Cambridge, USA \\ 2. Department of Organismic \& Evolutionary Biology, Harvard University, Cambridge, USA \\ 3. Department of Biological Anthropology, Harvard University, Cambridge, USA \\ 4. Center for Adaptive Behavior and Cognition, Max Planck Institute for Human Development, Lentzealle 94,14195 Berlin, \\ Germany \\ Corresponding author - Jeffrey R. Stevens
}

\begin{abstract}
The capacity for non-linguistic, numerical discrimination has been well characterized in non-human animals, with recent studies providing careful controls for nonnumerical confounds such as continuous extent, density, and quantity. More poorly understood are the conditions under which animals use numerical versus nonnumerical quantification, and the nature of the relation between these two systems. Here we test whether cotton-top tamarins and common marmosets can discriminate between two quantities on the basis of the amount of food rather than on number. In three experiments, we show that when choosing between arrays containing different numbers and sizes of food objects, both species based their decisions on the amount of food with only minor influences of numerical information. Further, we find that subjects successfully discriminated between two quantities differing by a 2:3 or greater ratio, which is consistent with the ratio limits found for numerical discrimination with this species. These studies demonstrate that non-human primates possess mechanisms that enable quantification of total amount, in addition to the numerical representations demonstrated in previous studies, with both types of quantification subject to similar processing limits.
\end{abstract}

Keywords: analog magnitude, callitrichids, foraging, non-linguistic quantification, numerical discrimination

\section{Introduction}

Over the past decades, a wealth of research has focused on the origins and nature of non-linguistic quantificational capacities in human and non-human species (reviewed in Boysen and Capaldi 1993; Boysen and Hallberg 2000; Brannon 2005b; Davis and Perusse 1988; Dehaene 1997; Feigenson et al. 2004; Hauser and Spelke 2004). Many of these studies have focused on relative numerosity-discriminations of which of two sets is larger (Anderson et al. 2005; Beran 2001; Call 2000; Menzel 1960; Rumbaugh et al. 1987; Thomas and Chase 1980; Uller et al. 2003) - and the cues used to discriminate stimuli, such as numerical distance, numerical ratio, item size, item orientation, etc. For instance, in a series of studies on the effect of stimulus properties on choice, Menzel $(1960,1961,1969)$ demonstrated that chimpanzees are very sensitive to differences in the size of food items (detecting $5 \%$ differences in length), but this sensitivity is modulated by the orientation of the food. These and other studies, however, typically have not directly assessed whether the test subjects use an amountbased cue for quantity, such as surface area and volume, or whether they use discrete number independent of amount. More importantly for the present work, even in cases which demonstrated amount-based discrimination, the processing limits of such discrimination have 
not been detailed and systematically compared with number-based discrimination. Where number-based discrimination has been explored, results generally parallel studies with human infants, revealing that a wide variety of non-human animals discriminate between visual-spatial arrays and auditory-temporal sequences on the basis of discrete number, both in studies that involve laboratory training (Boysen and Berntson 1989; Brannon and Terrace 1998; Matsuzawa 1985; Washburn and Rumbaugh 1991) and in studies testing spontaneous, untrained responses (Hauser et al. 1996, 2003; Lewis et al. 2005; Santos et al. 2005). In these cases, specific information has been provided about processing limits.

Numerical capacities in non-human animals seem to rely on two distinct mechanisms, revealing distinctive processing limits. First, animals can discriminate between approximate numerosities via a system of analog magnitudes, in which performance is limited by the ratio between the quantities independent of absolute value. Cantlon and Brannon (2006), for example, demonstrated that in operantly trained rhesus monkeys, ratio determined numerical discrimination between quantities ranging from 1 to 30 items. Human infants and adults also represent large approximate numbers and show similar signature ratio limits (Barth et al. 2003; Cantlon and Brannon 2006; Xu and Spelke 2000), suggesting a common system of numerical representation. Second, non-human animals can enumerate up to four objects precisely using a system of parallel individuation. Thus far, evidence for this system comes from studies of human adults, infants, and free-ranging rhesus monkeys (Macaca mulatta). For example, in a twobox choice study in which some number of objects were placed into one box, followed by the placement of some number of objects into a second box, rhesus monkeys successfully selected the larger number in comparisons of 1 versus 2, 2 versus 3 , and 3 versus 4 , but not in comparisons of 3 versus 8 or 4 versus 8 (Hauser et al. 2000). This pattern of results suggests that rhesus monkeys can store between three to four objects in working memory at one time (note that chimpanzees do successfully discriminate 3 vs. 8 and 4 vs. 8 when presented sequentially, Beran 2004; Beran and Beran 2004). Further, once those representations are established, individuals can perform different operations over those representations. Human infants, for instance, appear to base their decision on total amount rather than number; that is, when presented with one large cracker versus two mediumsized crackers that are equal in total amount to the large cracker, infants choose at chance. Similarly, when presented with one large cracker and two small crackers that are one-half of the total amount of the large cracker, infants prefer the one large cracker. Rhesus monkeys, on the other hand, appear to base their decision on number-when presented with one large apple slice and three apple slices equal in volume, rhesus prefer the container that received three slices. Although it remains untested whether rhesus can attend to total amount over number under these condition as do human infants, a recent study shows that rhesus attend to both number and total amount when presented with small numbers of non-solid collections (carrot pieces) that are poured from one container into another (Wood et al. 2007). The fact that human infants and adults demonstrate a similar limit in a variety of tasks (Feigenson et al. 2002; Luck and Vogel 1997; Pylyshyn and Storm 1988), again suggests continuity of this mechanism across ontogeny and phylogeny.

Some researchers have argued that using number may be a "last-resort" strategy of quantification (Breukelaar and Dalrymple-Alford 1998; Davis and Memmott 1982) when other cues such as amount cannot be used (but see Brannon 2005a). These mechanisms of quantification have presumably evolved to enhance survival and successful reproduction of individuals in their natural environment, such as in foraging, inter-group conflict, parental investment, and predator avoidance contexts (Hamilton 1971; Kitchen 2004; Lyon 2003; McComb et al. 1994; Wilson et al. 2001). Thus, the quantificational mechanism employed by animals should reflect the type of quantity information that is most relevant for the given context and the degree to which discrimination impacts fitness.

In foraging situations, for instance, animals often attempt to maximize the amount of food acquired per unit time spent foraging (Stephens and Krebs 1986). Though number frequently predicts total amount, it need not, particularly in circumstances in which the size of food items can differ greatly. Animals may often use non-numerical quantitative variables such as surface area and volume as the basis for discrimination (Davis and Perusse 1988), especially when the goal is to maximize amount (and not necessarily number), as in many foraging situations. In contrast, precisely discriminating number may be quite relevant for bird species vulnerable to brood parasitism in which tracking the addition of parasitic eggs is crucial (Lyon 2003). Importantly, the ability to numerically quantify egg number in these species does not imply the use of number in all contexts. That is, the presence of a numerical system does not preclude animals from using other cues such as amount to quantify objects in their environment.

The present study investigated whether cotton-top tamarins (Saguinus oedipus) and common marmosets (Callithrix jacchus) discriminate between two quantities on the basis of the amount of food rather than on number, and if so whether their pattern of performance reveals the set-size signature of parallel individuation (Feigenson et al. 2002; Hauser et al. 2000) or the ratio limit of the analog-magnitude system (Gallistel 1990). Furthermore, we examined the stability of these representations against other factors such as number and 
density. Specifically, we used a forced-choice task in which subjects selected and then consumed one of two arrays containing different numbers. Though many previous experiments using this technique to study relative numerosity assume that animals quantify based on amount, few have explicitly controlled for factors such as number and density. In Experiment 1, we presented food pieces of equal size to assess whether performance depended on the ratio between the comparison quantities or the three- to four-item limit of parallel individuation. In Experiments 2 and 3, we manipulated the numerical contrasts, density, and individual food size to determine the characteristics of the arrays on which the monkeys based their decisions.

Cotton-top tamarins were an obvious choice for these experiments as prior work provides evidence of numerical quantification, in which non-numerical parameters were systematically controlled (Hauser et al. 2002, 2003; Uller et al. 2001). In addition, comparative studies of both tamarins and marmosets suggest that they are sensitive to the relation between time and food quantity as evidenced by their patterns of both temporal and spatial discounting (Stevens et al. 2005a, b). For example, in one study the distance traveled for the larger food quantity was consistent for contrasts of 1 versus 3 and 2 versus 6 (Stevens et al. 2005b). Given these findings, we had strong a priori reasons to expect sensitivity to displays varying along the dimension of quantity.

\section{Experiment 1: \\ Analog magnitude versus parallel individuation}

In Experiment 1, we tested how subjects spontaneously responded to arrays of food rewards differing in the magnitude of reward amount and the numerical distance between those reward amounts (e.g., two vs. four pellets has a distance of two). We reasoned that when subjects can discriminate between the two arrays of food items, they should choose the larger array to maximize food intake; previously, we have demonstrated that tamarins do maximize their foraging rate in a self- control task (Stevens et al. 2005a). With this design, we tested between analog-magnitude and parallel-individuation systems of quantification by assessing discrimination abilities at various numerical ratios and magnitudes. The analog-magnitude system predicts that performance should vary as a function of the ratio between the comparison quantities as opposed to magnitudes, whereas parallel individuation predicts accurate performance for small magnitudes (one to three) and chance performance when one of the quantities exceeds the three- to four-item capacity limit.

\section{Methods}

\section{Subjects and apparatus}

Six cotton-top tamarins and six common marmosets (three males and three females of each species) of mixed experimental history participated in this experiment. We placed subjects in a triangular arena $(25 \times 25 \times 25 \times 37 \mathrm{~cm})$ facing the experimental apparatus (Figure 1a). The apparatus consisted of two food trays, each set on sliding tracks. When slid forward, subjects could reach through one of two small holes in the Plexiglas front of the arena to grasp a crossbar and draw the food tray forward. Each tray included a food bin consisting of black Tygon tubing cut in half lengthwise. We placed Research Associates $45 \mathrm{mg}$ purified primate diet banana-flavored pellets in holes (5 $\mathrm{mm}$ apart) drilled in the bottom of the tubing (Figure 1b).

\section{Experimental procedures}

To begin a trial, we blocked visual access to the food trays with an opaque barrier. We then placed the food into both bins simultaneously and arranged the pellets in a line. After loading the pellets, the experimenter removed the partition, looked down into his/her lap to avoid unintentionally cueing the subject, waited $5 \mathrm{~s}$, and simultaneously pushed both food trays forward to within the subject's reach. Subjects had $5 \mathrm{~s}$ to touch one of the crossbars and $30 \mathrm{~s}$ to consume the food. If either
Figure 1. (a) Experimental apparatus in which subjects drew one of two food bins forward to consume the pellets. In Experiment 1 , we arranged food pellets in a linear array (b), whereas in Experiments 2 and 3 , we placed food on a grid of either low density (c) or high density (d).

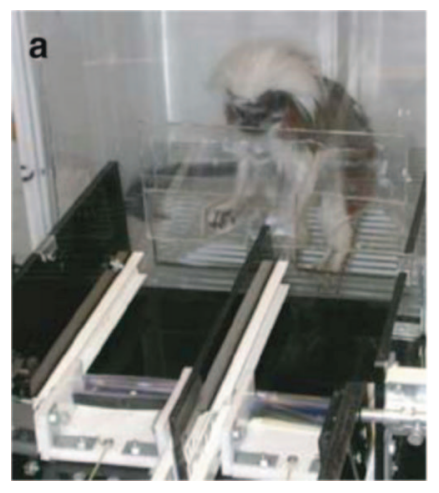

b

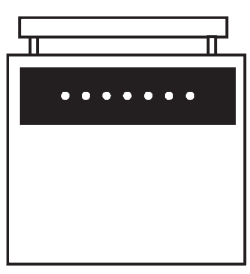

C

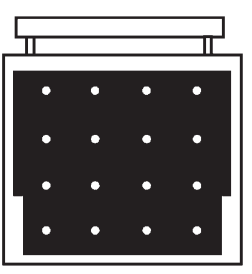

d

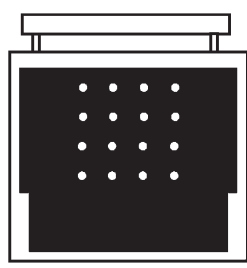


of these conditions was not met, we slid the trays back, removed the food, replaced the barrier, and marked the trials as 'no choice': out of 1,728 free choice trials completed in the test conditions, we scored only 14 as no choice. If the subject did touch one of the food trays, we immediately slid the opposing tray out of reach. After the subject acquired the last food pellet, we slid the tray back, removed the unconsumed food from the opposing food bin, and replaced the barrier. We then waited $5 \mathrm{~s}$ before loading the bins for the next trial. We recorded which side the subject pulled and the number of pellets received for each trial.

We tested subjects with 12 numerical pairs, using three different numerical distances and four magnitudes of the small reward, yielding numerical ratios (large/ small reward magnitude) ranging from 1.25 to 4.0 (Table 1). Each subject experienced 12 daily sessions of the 12 randomly ordered test trials plus four $(0,1)$ trials (one as the first trial and three randomly dispersed within the session) to verify motivation and attention to the sets; thus, each session consisted of a total of 16 trials.

\section{Training}

We trained subjects by allowing them to choose one of the two food trays and consume its contents. All subjects experienced training sessions consisting of 16 trials of a pseudorandom mixture of the following numerical pairs: $(0,1),(0,2),(0,3),(0,4),(1,5),(1,6)$, and $(1,7)$. The logic behind this design was to present extremely easy ratios at the start in order to train the animals on the basic setup. To complete training, we required subjects to select the smaller reward no more than twice in 12 trials for two consecutive sessions. If in the experimental sessions a subject selected the 0 reward twice, we aborted the session and ran a training session in the subsequent session (two tamarins experienced this type of retraining). If in the experimental sessions the subject selected the small reward five or more times and four or more of these were on the same side, we classified their performance as mediated by a side bias and moved to a

Table 1. Numerical pairs and ratios as a function of numerical distance and magnitude of smallest reward

\begin{tabular}{lllll}
\hline \multicolumn{5}{c}{ Small magnitude } \\
\cline { 2 - 5 } \multicolumn{1}{c}{2} & 3 & 4 \\
\hline Numerical distance \\
1 & $(1,2) 2.0$ & $(2,3) 1.5$ & $(3,4) 1.33$ & $(4,5) 1.25$ \\
2 & $(1,3) 3.0$ & $(2,4) 2.0$ & $(3,5) 1.67$ & $(4,6) 1.5$ \\
3 & $(1,4) 4.0$ & $(2,5) 2.5$ & $(3,6) 2.0$ & $(4,7) 1.75$
\end{tabular}

Numbers in parentheses are numerical pairs, and numbers to the right are numerical ratios corrective measure in the next session (two tamarins experienced a side bias). Specifically, we ran a training session focusing on the opposite side as their bias. To move from these training sessions back to an experimental session, the subjects could make no more than two mistakes (choose small reward) in a single training session.

\section{Statistical analysis}

We analyzed the data with a series of repeated-measures analyses of variance (ANOVA). For our dependent variable, we used the arcsine, square-root transformed proportion of choices for the larger reward in each session for each subject. In the first analysis we used numerical ratio as a within-subjects factor and species as a between-subjects factor. We pooled data from numerical pairs with the same ratios (pooling did not alter the results). Other analyses examined effects of trials and sessions in a similar manner. Assumptions of sphericity were not violated in any of the analyses. We report Bonferroni-corrected individual contrasts for pairwise comparisons.

\section{Results and discussion}

Numerical ratio significantly influenced choice behavior $(F(8,80)=10.14, P<0.01)$, resulting in a strong preference for the larger amount at high ratios (Figure 2). The two species did not differ in the proportion of trials in which they chose the larger amount (marmoset mean $=0.72, \mathrm{SE}=0.17$; tamarin mean $=0.67$, $\mathrm{SE}=0.17 ; F(1,10)=0.94, P=0.35)$. Neither trial number $(F(14,140)=0.91, P=0.55)$ nor session $(F(11,110)=1.60$, $P=0.11)$ influenced choice, suggesting little effect of learning on preferences. Due to the absence of a species effect in the ANOVA, we collapsed the data across species. Because performance varied as a function of ratio (a signature of the analog-magnitude system), we conducted a series of nine one-sample $t$-tests to determine whether the mean response at each ratio differed from chance levels of choice (0.5). Using a Bonferroni correction, statistically significant $P$-values could not exceed $a=0.05 / 9=0.0056$. Based on this criterion, subjects preferred larger rewards at ratios of 1.5 and greater (Figure 2). Therefore, as demonstrated in other primates species (Anderson et al. 2004; Beran 2001; Boysen and Berntson 1989, 1995; Brannon and Terrace 2002; Call 2000; Rumbaugh et al. 1987; Shumaker et al. 2001), tamarins and marmosets show a preference for the larger of two sets of food items and show an effect of the ratio between sets on preference.

We found no evidence that the three- to four-item capacity limit of parallel individuation influenced the monkeys' performance under these testing conditions. In cases in which multiple numerical pairs shared the same ratio but spanned this numerical limit $([2,3]$ and 


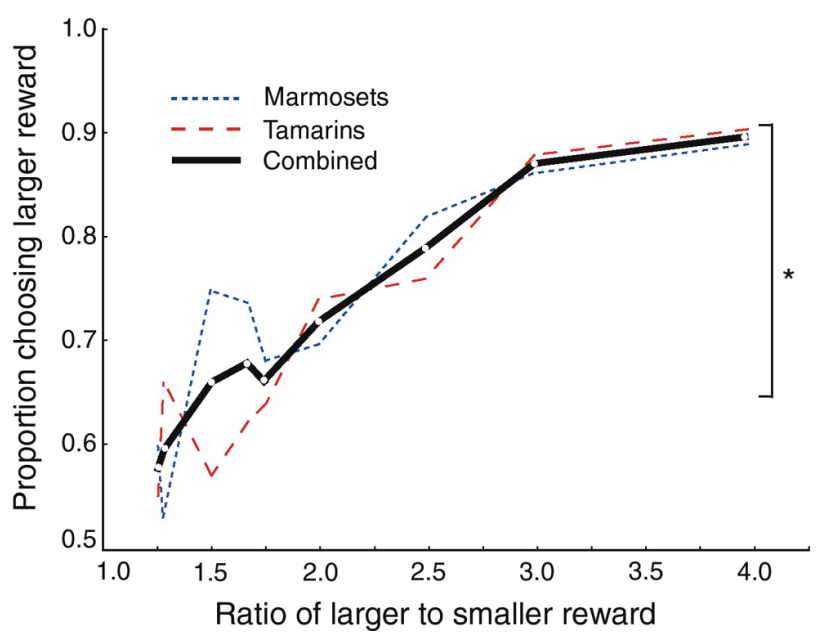

Figure 2. Choice as a function of ratio of large to small reward magnitude.

$[4,6]$ or $[1,2]$ and $[2,4]$ and $[3,6])$, individual contrasts (Bonferroni-corrected $a=0.05 / 4=0.0125$ ) showed no differences within ratios, suggesting that the monkeys did not use parallel individuation to quantify at small magnitudes.

\section{Experiment 2: Number versus amount}

Results from Experiment 1 indicate that both tamarins and marmosets rely on analog-magnitude representations when choosing between two visible arrays of food. It is not clear from these data, however, whether the subjects attended to the number of food rewards or the continuous amount of food available (surface area, volume, etc.). Experiment 2 provides a test of this question by parametrically varying the relationship between food amount and number, as well as the density of food rewards. If subjects use only number, they should always choose the reward with the larger numerical magnitude. If, however, they attend only to amount, subjects should always choose the reward with the greatest total surface area or volume.

\section{Methods}

Because the tamarins and marmosets did not differ in their preferences in Experiment 1, and due to other lab-related constraints at the time of test, we focused on cotton-top tamarins (five subjects) in Experiments 2 and 3. We used a similar apparatus to Experiment 1; however, instead of placing a linear array of food pellets in a trough, we randomly placed the food pellets on a $4 \times 4$ Plexiglas grid (Figure 1c, d). Each subject had $5 \mathrm{~s}$ to choose an option by drawing one of two trays forward. If subjects did not consume all of the food after $15 \mathrm{~s}$ or if they dropped food pellets, the experimenter hand-fed unconsumed pellets to the subjects.

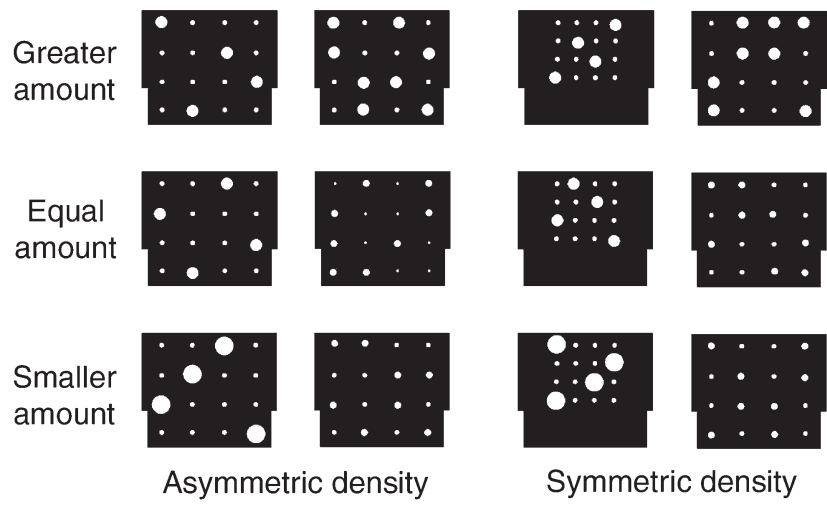

Figure 3. Food pellet presentation for Experiment 2. The larger number of items was associated with either a greater, equal, or smaller amount of food (see text), and the larger number was either more dense than the smaller number (asymmetric) or equally dense (symmetric). Pellet size is not to scale.

We tested subjects using a factorial combination of three experimental conditions: numerical pair, number/ amount correlation, and density (Figure 3). We tested two numerical pairs defined by the same ratio difference but spanning the numerical limit of parallel individuation: $(1,2)$ and $(4,8)$.

To determine whether the monkeys discriminated number or amount, we varied the correlation between these two parameters by using differently sized food pellets (Figure 3); therefore, the number of items was associated with either a greater, equal, or smaller amount of food. In the greater amount condition, the option with the larger number also had the greater total amount. We used medium-sized pellets (the same size as in Experiment $1-45 \mathrm{mg}$ ) for both food trays. In the equal amount condition, the options differed in number but provided the same total amount of food. The larger number option had small pellets $(20 \mathrm{mg})$ and the smaller number option had medium pellets (45 mg), so amount was equated across choices. For the smaller amount condition, the array with the larger number of food items contained the smaller total food amount, because the array with the larger number consisted of small food pellets (20 mg) while the array with the smaller number consisted of large food pellets $(90 \mathrm{mg})$.

We manipulated density of the food pellets by varying the distance between possible food positions in the grid. In the asymmetric condition, we placed the pellets in equally spaced holes (12 $\mathrm{mm}$ between the holes) for both the larger and smaller options. In the symmetric condition, we used the $12 \mathrm{~mm}$ distance between holes for the larger number of pellets (Figure 1c) and a $6 \mathrm{~mm}$ distance between holes for the smaller number of pellets (Figure 1d). Therefore, in the asymmetric case, the array with the larger number had a higher density than the smaller number, whereas in the symmetric case, the density was approximately equal for both amounts. 
We tested subjects with one trial of each of the 12 possible conditions in one session. We randomly ordered the trial conditions and sides in each of 12 sessions, but all subjects experienced the same ordering per session.

\section{Results and discussion}

In a repeated-measures ANOVA using numerical pair, number/amount correlation, and density as factors, only the correlation factor significantly influenced choice $(F(2,8)=3.46, P<0.01)$. All three correlation conditions differed from each other (Bonferroni-corrected individual contrasts, $a=0.05 / 3=0.017)$, resulting in subjects choosing the larger number significantly more often in the greater amount treatment than in the equal amount treatment and choosing the larger number more in the equal amount treatment than in the smaller amount treatment (Figure 4). Recall that in the smaller amount condition, the smaller number of pellets had a greater amount of food. Thus, the tamarins' preference for the smaller number suggests that they attended to amount over number. In the equal amount condition, the tamarins showed a bias towards the smaller number even when both arrays contained equal amounts of food. This deviation from the prediction of indifference could result from either increased handling required to consume many smaller pellets or a preference for the more monopolizable larger pellets.

All two-way interactions showed significant effects (number $\times$ density: $F(1,4)=9.24, P=0.04$; number $\times$ correlation: $F(2,8)=6.43, P=0.02$; correlation $\times$ density:

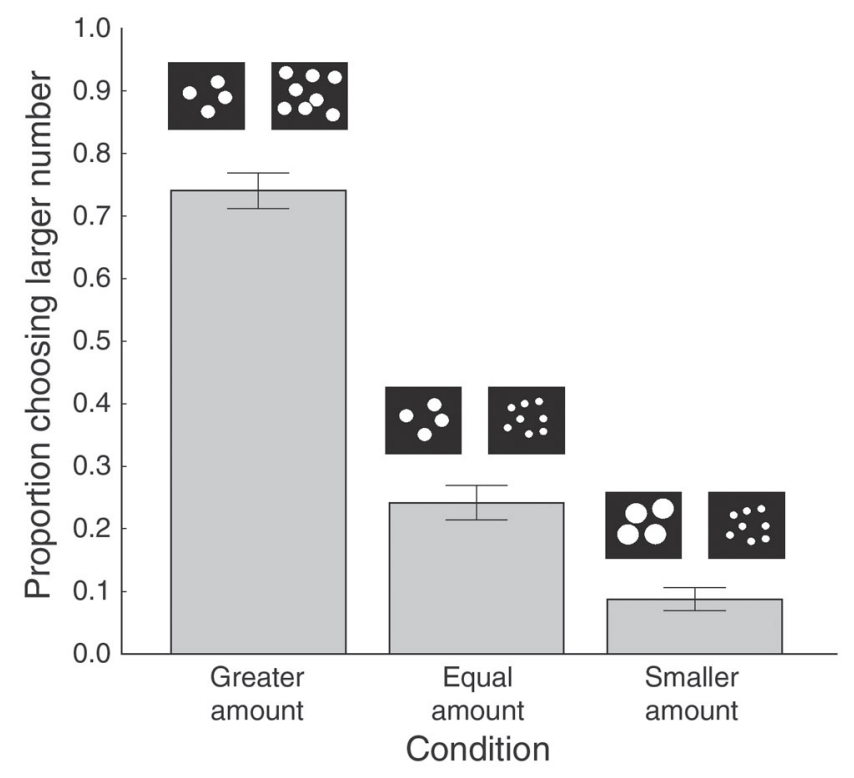

Figure 4. Preference for larger number as a function of whether number was associated with either a greater, equal, or smaller amount of food in Experiment 2. Error bars represent the standard error of the mean. Pellet size is not to scale.
$F(2,8)=6.70, P=0.02)$. The number $\times$ density interaction is likely spurious because density cannot be defined with a single item. Though density did not influence choice in the 1 versus 2 condition, there was a trend for a preference for the more dense option in the 4 versus 8 condition. The number $\times$ correlation interaction results from a slightly increased preference for the larger number in the 4 versus 8 pair compared to the 1 versus 2 pair in the smaller amount condition (Bonferroni-corrected individual contrast, $a=0.05 / 12=0.004)$. Thus, numerical information has somewhat more of an influence on discrimination at greater magnitudes when number is pitted against total amount. Finally, in the correlation $\times$ density interaction, subjects tended to prefer the more dense option in the equal amount condition. Because of these possible effects on discrimination, we further explored the role of density in Experiment 3.

\section{Experiment 3: Density and amount}

In this experiment, we investigated the role of density information in quantification of total amount by holding the number of food items constant in all pairs, while varying the density and size of food pellets.

\section{Methods}

As in Experiment 2, tamarin subjects chose between two grids of food pellets. We tested subjects in three conditions, all with four versus four food pellets (Figure 5). Five subjects experienced six sessions of nine trials (three replicates of the three conditions). In the same/asymmetric condition, the amount of food remained constant across choices but the density varied. In this condition, both sets of four pellets were the same size (medium, $45 \mathrm{mg}$ pellets) and the density was asymmetric between the two choices (one option on the $12 \mathrm{~mm}$ grid and the other on the $6 \mathrm{~mm}$ grid). In the different/asymmetric condition, we used differently sized food pellets (medium, $45 \mathrm{mg}$ and large, $90 \mathrm{mg}$ ) and different densities, such that the large pellets were more dense than the medium pellets. We did not conduct a condition in which the larger pellets were arranged more sparsely, because given the previously demonstrated effect of pellet size, size likely would swamp the effects of density. In the different/symmetric condition, the subjects chose between differently sized pellets of the same density.

\section{Results and discussion}

Subjects showed a clear preference for the more dense option even when holding number and amount constant (same/asymmetric condition-one-sample $t$-test: 


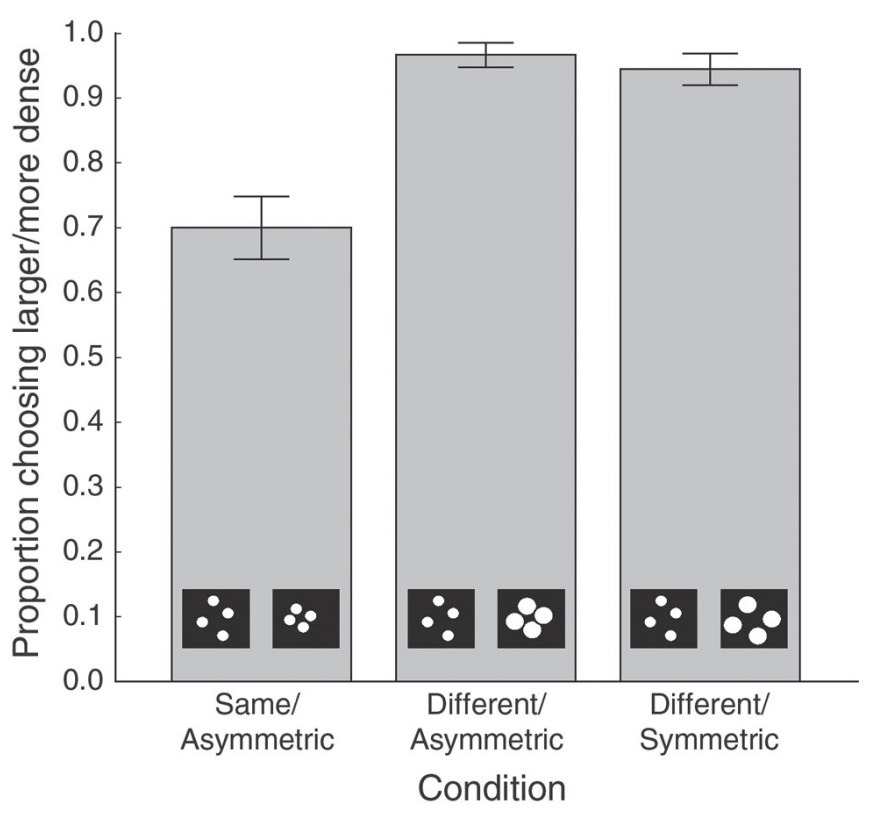

Figure 5. Preference for larger amount or more dense food items as a function of density and food size differential in Experiment 3. Error bars represent the standard error of the mean. Pellet size is not to scale.

$t_{4}=4.43, \mu=0.5, P=0.01$ ), suggesting that density influences quantification of total amount (Figure 5). Subjects showed an even stronger preference for the larger amount when number was held constant, regardless of density (repeated-measures ANOVA: $F_{2,8}=38.55$, $P<0.01)$. Therefore, the tamarins attended to the amount of food material when choosing among options with equivalent numbers of food items, and the effect of amount trumped any effect of density on preferences.

\section{General discussion}

The present studies investigated the mechanisms supporting non-human animals' capacity to quantify over sets of food items on the basis of total amount. In Experiment 1, cotton-top tamarins and common marmosets chose the array containing the greater total amount of food or number of food items, with performance varying as a function of the ratio between the comparison quantities. Experiment 2 showed that tamarins based their decision on the total amount of food rather than on the number of food items when number and total amount systematically varied across the two arrays. Experiment 3 extended these findings by showing that tamarins continued to choose the array with the greater amount of food even when number was equated between the conditions. Experiments 2-3 further revealed that quantitative factors such as the density or size of the items in the array can influence representations of total amount.
These studies provide three contributions to our understanding of the origins and nature of non-linguistic representations of quantity. First, these studies provide the first systematic investigation showing that non-human animals quantify over sets on the basis of total amount of food rather than number. Again, many other studies have assumed that animals quantify using amount and even demonstrated that non-numerical features such as item size and orientation influence quantity discrimination (Anderson et al. 2005; Beran 2001; Call 2000; Menzel 1960; Rumbaugh et al. 1987; Thomas and Chase 1980; Uller et al. 2003). These studies, however, have not excluded the possibility that animals could combine amount and number information to judge quantity. Our results suggest that tamarins, and presumably other animals, can construct representations of total amount with little to no influence from numerical information. Though this may appear unsurprising, it is not clear that this is the case for humans. Infants are more likely to notice changes in number amidst varying total amount as opposed to changes in total amount amidst varying number (Brannon et al. 2004); similarly, adults cannot ignore number information when making judgments about total amount (Barth 2003), with arrays containing greater numbers falsely judged to contain a greater total amount. Thus for tamarins, but not humans, representations of total amount and number appear to be nearly independent. Along these lines, and as pointed out in Experiment 2, tamarins preferred the less numerous array when amount was equated, suggesting a shift toward other factors in a foraging context. For example, perhaps individuals pick larger packets of food because these are easier to handle or easier to monopolize under certain contexts. It will thus be essential to work out experimental procedures on animals that avoid using food, and symmetrically, run experiments with humans that explicitly target food.

Second, tamarins use numerical and non-numerical information differently, depending upon context. Hauser et al. (2003) demonstrated that tamarins spontaneously discriminated between auditory sequences on the basis of number, and Uller et al. (2001) showed that tamarins use numerical cues to discriminate mathematical operations such as addition (e.g., $1+1=2$ vs. $1+1=3$ ) over food items. Therefore, tamarins have the ability to assess discrete number, in contexts with and without food. Our current findings, however, suggest that instead tamarins use relative amount in some circumstances. These differences likely result from methodological differences. In the current study, the subjects faced a simultaneous choice task with both stimuli constantly in view, and thus they could compare the arrays directly and on the basis of perceptual cues. In contrast, in the previous studies the stimuli (either auditory or visual) were presented sequentially, requiring the subjects to store representations of the target items in mem- 
ory. Undoubtedly, animals can use both number and amount to quantify items in their environment (Brannon 2005a; Davis and Perusse 1988). Our work suggests that we need to explore the conditions by which each of these mechanisms is engaged. It remains unclear how aspects of the environment such as the task (forced choice, expectancy violation), the modality (visual, auditory), demands (in full view versus stored in memory), and the domain (food, communication, predator avoidance, mating opportunities) influence the implementation of these mechanisms.

Finally, our studies provide insight into the nature of the processes operating over numerical and non-numerical quantification. Tamarins attend to number in some contexts and to total amount in other contexts. Thus, at some level, distinct processes pick out unique properties of a group depending on the task. However, once numerical or non-numerical information has been computed, these values may be stored in a common mental mechanism. Our results from Experiment 1 show that tamarins successfully chose the array with the greater amount of food, provided that the two sets differed by a 2:3 ratio. Likewise, in Hauser et al. (2003) tamarins spontaneously discriminated number for comparisons that differed by a 2:3 ratio (4 vs. 6,8 vs. 12 ) but not by a $4: 5$ ratio ( 4 vs. 5,8 vs. 10 ). Therefore, tamarins show a similar ratio limit when comparing sets on the basis of numerical and non-numerical factors, suggesting that the same mental mechanism may underlie the ability to retain information about number and total amount. Thus, similar to previous claims suggesting that common processes support the capacity to measure temporal duration and to represent number (Gallistel 1990; Meck and Church 1983; vanMarle and Wynn 2006), we suggest that common processes support the capacities to represent number and total amount. Future studies could further explore this possibility by testing whether other similarities also exist between non-human primates' representation of number and amount.

Acknowledgments - We would like to thank NIH (NRSA) for funding J.R.S., Harvard University and NIH (NRSA) for funding J.N.W., and NSF (ROLE) for funding M.D.H. We would also like to thank David Glynn, Ian Goh, Sarah Heilbronner, Jeff Lau, Cori McLean, Alex Rosati, and Amy Tao for assistance in conducting this experiment. We are grateful to three anonymous referees for helpful comments on the manuscript. This experiment was approved by the Institutional Animal Care and Use Committee at Harvard University (Animal Subjects Codes 92-16 and 22-07) and conforms to the APA Guidelines for Ethical Conduct in the Care and Use of Animals.

\section{References}

Anderson JR, Awazu S, Fujita K (2004) Squirrel monkeys (Saimiri sciureus) choose smaller food arrays: long-term retention, choice with nonpreferred food, and transposition. J Comp Psychol 118:58-64

Anderson US, Stoinski TS, Bloomsmith MA, Marr MJ, Smith AD, Maple TL (2005) Relative numerousness judgment and summation in young and old Western lowland gorillas. J Comp Psychol 119:285-295

Barth H (2003) Discrete and continuous cues to quantity in large sets of food items. Paper presented at the annual meeting of the Psychonomic Society, Vancouver

Barth H, Kanwisher N, Spelke ES (2003) The construction of large number representations in adults. Cognition 86:201-221

Beran MJ (2001) Summation and numerousness judgments of sequentially presented sets of items by chimpanzees (Pan troglodytes). J Comp Psychol 115:181-191

Beran MJ (2004) Chimpanzees (Pan troglodytes) respond to nonvisible sets after one-by-one addition and removal of items. J Comp Psychol 118:25-36

Beran MJ, Beran MM (2004) Chimpanzees remember the results of one-by-one addition of food items to sets over extended time periods. Psychol Sci 15:94-99

Boysen ST, Berntson GG (1989) Numerical competence in a chimpanzee (Pan troglodytes). J Comp Psychol 103:23-31

Boysen ST, Berntson GG (1995) Responses to quantity: perceptual versus cognitive mechanisms in chimpanzees (Pan troglodytes). J Exp Psych Anim B 21:82-86

Boysen ST, Capaldi EJ (1993) The development of numerical competence: animal and human models. Lawrence Erlbaum, Hillsdale

Boysen ST, Hallberg KI (2000) Primate numerical competence: contributions toward understanding nonhuman cognition. Cog Sci 24:423

Brannon EM (2005a) The numerical ability of animals. In: Campbell JID (eds) Handbook of mathematical cognition. Psychology Press, New York, pp 85-108

Brannon EM (2005b) Quantitative thinking: from monkey to human and human infant to adult. In: Dehaene $S$, Duhamel J-R, Hauser MD, Rizzolatti G (eds) From monkey brain to human brain. MIT, Cambridge, pp 97-116

Brannon EM, Abbott S, Lutz DJ (2004) Number bias for the discrimination of large visual sets in infancy. Cognition 93:B59-B68

Brannon EM, Terrace HS (1998) Ordering of the numerosities 1 to 9 by monkeys. Science 282:746-749

Brannon EM, Terrace HS (2002) The evolution and ontogeny of ordinal numerical ability. In: Bekoff $M$, Allen C, Burghardt GM (eds) The cognitive animal: empirical and theoretical perspectives on animal cognition. MIT, Cambridge, pp 197-204

Breukelaar JW, Dalrymple-Alford JC (1998) Timing ability and numerical competence in rats. J Exp Psych Anim B 24:84-97

Call J (2000) Estimating and operating on discrete quantities in orangutans (Pongo pygmaeus). J Comp Psychol 114:136-147 
Cantlon JF, Brannon EM (2006) Shared system for ordering small and large numbers in monkeys and humans. Psychol Sci 17:401-406

Davis H, Memmott J (1982) Counting behavior in animals: a critical evaluation. Psychol Bull 92:547-571

Davis H, Perusse R (1988) Numerical competence: from backwater to mainstream of comparative psychology. Behav Brain Sci 8:47-68

Dehaene S (1997) The number sense. Oxford University Press, New York

Feigenson L, Carey S, Hauser MD (2002) The representations underlying infants' choice of more: object files versus analog magnitudes. Psychol Sci 13:150-156

Feigenson L, Dehaene S, Spelke ES (2004) Core systems of number. Trends $\operatorname{Cog}$ Sci 8:307-314

Gallistel CR (1990) The organization of learning. MIT, Cambridge

Hamilton WD (1971) Geometry for the selfish herd. J Theor Biol 31:295-311

Hauser MD, Carey S, Hauser LB (2000) Spontaneous number representation in semi-free-ranging rhesus monkeys. Proc R Soc Lond B 267:829-833

Hauser MD, Dehaene S, Dehaene-Lambertz G, Patalano A (2002) Spontaneous number discrimination of multi-format auditory stimuli in cotton-top tamarins (Saguinus oedipus). Cognition 86:B23-B32

Hauser MD, MacNeilage P, Ware M (1996) Numerical representation in primates. Proc Nat Acad Sci USA 93:1514-1517

Hauser MD, Spelke ES (2004) Evolutionary and developmental foundations of human knowledge: a case study of mathematics. In: Gazzaniga M (ed) The cognitive neurosciences III. MIT, Cambridge, pp 853-864

Hauser MD, Tsao F, Garcia P, Spelke ES (2003) Evolutionary foundations of number: spontaneous representation of numerical magnitudes by cotton-top tamarins. Proc R Soc Lond B 270:1441-1446

Kitchen DM (2004) Alpha male black howler monkey responses to loud calls: effect of numeric odds, male companion behaviour and reproductive investment. Anim Behav 67:125-139

Lewis KP, Jaffe S, Brannon EM (2005) Analog number representations in mongoose lemurs (Eulemur mongoz): evidence from a search task. Anim Cog 8:247-252

Luck SJ, Vogel EK (1997) The capacity of visual working memory for features and conjunctions. Nature 390:279-281

Lyon BE (2003) Egg recognition and counting reduce costs of avian conspecific brood parasitism. Nature 422:495-499

Matsuzawa T (1985) Use of numbers by a chimpanzee. Nature 315:57-59

McComb K, Packer C, Pusey AE (1994) Roaring and numeri$\mathrm{cal}$ assessment in contests between groups of female lions, Panthera leo. Anim Behav 47:379-387

Meck WD, Church RM (1983) A mode control model of counting and timing processes. J Exp Psych Anim B 9:320-334
Menzel EW (1960) Selection of food by size in the chimpanzee, and comparison with human judgments. Science 131:1527-1528

Menzel EW (1961) Perception of food size in chimpanzee. J Comp Physiol Psych 54:588-591

Menzel EW (1969) Responsiveness to food and signs of food in chimpanzee discrimination learning. J Comp Physiol Psych 68:484-489

Pylyshyn ZW, Storm RW (1988) Tracking multiple independent targets: evidence for a parallel tracking mechanism. Spat Vis 3:179-197

Rumbaugh DM, Savage-Rumbaugh S, Hegel MT (1987) Summation in the chimpanzee (Pan troglodytes). J Exp Psych Anim B 13:107-115

Santos LR, Barnes JL, Mahajan N (2005) Expectations about numerical events in four lemur species (Eulemur fulvus, Eulemur mongoz, Lemur catta and Varecia rubra). Anim Cog 8:253-262

Shumaker RW, Palkovich AM, Beck BB, Guagnano GA, Morowitz H (2001) Spontaneous use of magnitude discrimination and ordination by the orangutan (Pongo pygmaeus). J Comp Psychol 115:385-391

Stephens DW, Krebs JR (1986) Foraging theory. Princeton University Press, Princeton

Stevens JR, Hallinan EV, Hauser MD (2005a) The ecology and evolution of patience in two New World monkeys. Biol Lett 1:223-226

Stevens JR, Rosati AG, Ross KR, Hauser MD (2005b) Will travel for food: spatial discounting in two New World primates. Curr Biol 15:1855-1860

Thomas RK, Chase L (1980) Relative numerousness judgments by squirrel monkeys. Bull Psychon Soc 16:79-82

Uller C, Hauser MD, Carey S (2001) Spontaneous representation of number in cotton-top tamarins (Saguinus oedipus). J Comp Psychol 115:248-257

Uller C, Jaeger R, Guidry G, Martin C (2003) Salamanders (Plethodon cinereus) go for more: rudiments of number in an amphibian. Anim Cog 6:105-112

vanMarle K, Wynn K (2006) 6-month-old infants use analog magnitudes to represent duration. Dev Sci 9:F41-F49

Washburn DA, Rumbaugh DM (1991) Ordinal judgments of numerical symbols by macaques (Macaca mulatta). Psychol Sci 2:190-193

Wilson ML, Hauser MD, Wrangham RW (2001) Does participation in intergroup conflict depend on numerical assessment, range location or rank for wild chimpanzees? Anim Behav 61:1203-1216

Wood JN, Hauser MD, Glynn DD, Barner D (2007) Free-ranging rhesus monkeys spontaneously individuate and enumerate small numbers of nonsolid portions. Cognition (in press)

Xu F, Spelke ES (2000) Large number discrimination in 6-month-old infants. Cognition 74:B1-B11 